\title{
Relocation of genes generates non-conserved chromosomal segments in Fusarium graminearum that show distinct and co-regulated gene expression patterns
}

Chunzhao Zhao ${ }^{1,2,3,4}$, Cees Waalwijk ${ }^{1,2}$, Pierre JGM de Wit ${ }^{2,5}$, Dingzhong Tang ${ }^{3}$ and Theo van der Lee (2, $^{*}$

\begin{abstract}
Background: Genome comparisons between closely related species often show non-conserved regions across chromosomes. Some of them are located in specific regions of chromosomes and some are even confined to one or more entire chromosomes. The origin and biological relevance of these non-conserved regions are still largely unknown. Here we used the genome of Fusarium graminearum to elucidate the significance of non-conserved regions.
\end{abstract}

Results: The genome of $F$. graminearum harbours thirteen non-conserved regions dispersed over all of the four chromosomes. Using RNA-Seq data from the mycelium of F. graminearum, we found weakly expressed regions on all of the four chromosomes that exactly matched with non-conserved regions. Comparison of gene expression between two different developmental stages (conidia and mycelium) showed that the expression of genes in conserved regions is stable, while gene expression in non-conserved regions is much more influenced by developmental stage. In addition, genes involved in the production of secondary metabolites and secreted proteins are enriched in non-conserved regions, suggesting that these regions could also be important for adaptations to new environments, including adaptation to new hosts. Finally, we found evidence that non-conserved regions are generated by sequestration of genes from multiple locations. Gene relocations may lead to clustering of genes with similar expression patterns or similar biological functions, which was clearly exemplified by the PKS2 gene cluster.

Conclusions: Our results showed that chromosomes can be functionally divided into conserved and non-conserved regions, and both could have specific and distinct roles in genome evolution and regulation of gene expression.

Keywords: Gene expression, Non-conserved region, Gene relocation, Secondary metabolite gene cluster, Fusarium graminearum

\section{Background}

The genus Fusarium includes a large group of phytopathogenic fungi, each having a different or partially overlapping host range. F. graminearum is an important pathogen on wheat, barley and maize, causing Fusarium head blight $[1,2]$, while $F$. verticillioides mainly infects maize, causing rot and wilting [3]. In contrast, F. oxysporum causes disease on more than 100 different plant species, but specific

\footnotetext{
* Correspondence: theo.vanderlee@wur.nl

'Plant Research International, Wageningen, The Netherlands

${ }^{2}$ Graduate School Experimental Plant Sciences, Wageningen University,

Wageningen, The Netherlands

Full list of author information is available at the end of the article
}

strains usually infect only a single host species [4]. This has led to the introduction of the forma specialis concept in $F$. oxysporum, where strains are categorized according to the host plant they infect, such as tomato or banana [5]. In addition, some strains of $F$. oxysporum may also cause infections in humans [6]. Genomic sequences of several Fusarium species have been generated, including F. graminearum (strain PH-1), F. verticillioides (strain FGSC 7600), F. pseudograminearum (strain CS3096), F. solani (strain FGSC 9596), F. fujikuroi (strain IMI58289) and F. oxysporum f. sp. lycopersici strain 4287. In addition, 11 different $F$. oxysporum strains, including pathogens from 
different hosts as well as a biocontrol strain have been sequenced [7-11]. Genome comparisons showed that chromosome XII of $F$. fujikuroi is absent in the genome sequence of related species $F$. verticillioides, while 285 and $820 \mathrm{~kb}$ genome sequences at both ends of chromosome IV of $F$. verticillioides are absent in F. fujikuroi [11]. Comparing the genome of F. pseudograminearum with F. graminearum showed that $89.8 \%$ of genomic sequence could be aligned at $>70 \%$ nucleotide identity [7]. Strikingly, $F$. oxysporum 4287 includes 11 core chromosomes and four lineage-specific (LS) chromosomes. LS chromosomes are specific to $F$. oxysporum strain 4287 and have no collinear chromosomes in either F. graminearum, F. verticillioides or other $F$. oxysporum strains. The LS chromosomes in F. oxysporum 4287 are suggested to have resulted from horizontal transfer from unknown sources [9]. On LS chromosome 14, several effector-encoding genes have been identified that facilitate infection of its host plant [12-14].

In contrast to the high number of chromosomes present in F. verticillioides (11), F. oxysporum (15) and F. fujikuroi (12), F. graminearum has only four chromosomes, which probably resulted from fusions of ancestral chromosomes [8]. Comparing the genome of F. graminearum isolate PH-1 with F. graminearum isolate GZ3639 revealed several regions with high SNP density [8]. In addition, comparison of the genome of F. graminearum with the closely related species $F$. verticillioides and $F$. oxysporum revealed several non-conserved regions [9]. Further analysis showed that high SNP density regions match with non-conserved regions. Although many of the genes specifically expressed during plant infection are enriched in non-conserved regions [8], the origin and the biological relevance for these non-conserved regions are still largely unknown. In this study, we explored RNA-Seq data from both conidia and mycelium of $F$. graminearum to investigate the putative effect of gene locations (in conserved or non-conserved regions) on their expression patterns. In addition, by comparing the genome of $F$. graminearum with those of $F$. verticillioides and $F$. oxysporum, we show a possible mechanism for the generation of non-conserved regions.

\section{Results}

Synteny block analysis between the genomes of Fusarium graminearum, F. verticillioides and F. oxysporum.

To identify collinear regions on the chromosomes of F. graminearum $(\mathrm{Fg})$ and $F$. verticillioides $(\mathrm{Fv})$, we performed synteny block analysis presented in Figure 1. This analysis revealed that $F$. graminearum chromosome 1 (Fgchr 1 ) is largely collinear with $F$. verticillioides chromosomes (Fvchrs) 1, 5 and 8; Fgchr 2 is collinear with Fvchrs 6, 9, 10 and 11; Fgchr 3 is collinear with Fvchrs 2, 4 and 7; while Fgchr 4 is largely collinear with Fvchrs 2 and 3 (Figure 1A). These major collinear regions between chromosomes indicate that the four $F$. graminearum chromosomes were likely formed primarily through endto-end joining of two to four smaller ancestral chromosomes, descendants of which still exist in F. verticillioides. Similar collinear patterns were observed when comparing the genome of $F$. graminearum with those of $F$. oxysporum (Figure 1B). In addition to these major collinear regions, translocations and inversions of many chromosomal segments are observed in the chromosomes of F. graminearum. For instance, the first half of Fgchr 1 is homologous to Fvchr 1, but the order of genomic sequences on Fgchr 1 is different from Fvchr 1. Some chromosomal regions are translocated to new positions, while some are inversed (Figure 1A, C). In addition, thirteen non-conserved regions, which were designated $\mathrm{Nc1-Nc13}$, were found on the four chromosomes of F. graminearum. These non-conserved regions exhibit almost no synteny with the chromosomes of $F$. verticillioides (Figure 1C). Notably, although the second half of Fgchr 2 shows collinearity with Fvchrs 10 and 11, a large number of small synteny blocks was identified, indicating multiple rearrangements in this part of chromosome 2 (Figure 1A, C). Similar chromosome rearrangement patterns were observed when the chromosomes of F. graminearum were compared with those of F. oxysporum 4287 (Figure 1B). We also performed synteny block analysis between $F$. oxysporum and $F$. verticillioides. Consistent with the notion that F. oxysporum and F. verticillioides are more closely related to each other than to F. graminearum, the collinearity between $F$. oxysporum and $F$. verticillioides was much higher (Additional file 1).

\section{Gene expression analysis on individual chromosomes of F. graminearum}

To investigate a possible correlation between chromosome rearrangements and gene expression patterns on each chromosome of F. graminearum, we performed RNA-Seq analysis on mycelium of wild-type isolate $\mathrm{PH}-1$ grown in nutrient-rich medium. Three biologically independent replicates were conducted. In total, 35,103,060 RNA-Seq reads were mapped against the four chromosomes and the expression level of each chromosome was evaluated by using RPKM (reads per kilobase per million mapped reads) values. The averaged expression levels of genes on chromosome 1 are significantly higher than other chromosomes, while the expression levels of genes on chromosome 2 are significantly lower (Figure 2A). Subsequently, we evaluated the expression levels of all predicted genes of F. graminearum (Additional file 2) and divided them into five categories based on their RPKM values: RPKM 0, RPKM 0-1, RPKM 1-10, RPKM 10-100 and RPKM >100. The proportion of each category on each chromosome was calculated. As expected, the proportion of weakly expressed genes (e.g. RPKM <1) is higher on Fgchr 2 (45.1\%) than on the other chromosomes (30.2-35.3\%) (Figure 2B), while the proportion of highly expressed genes 


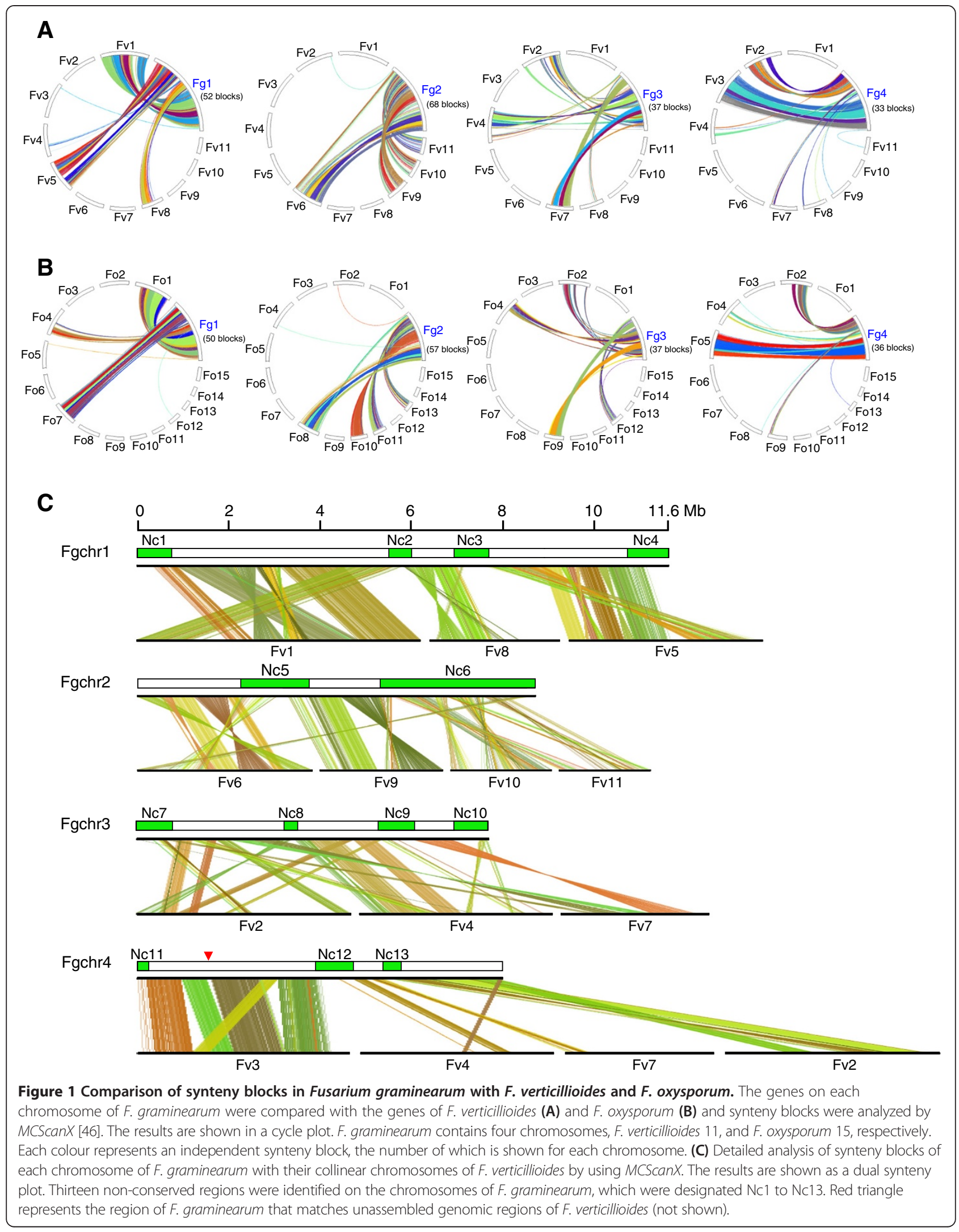



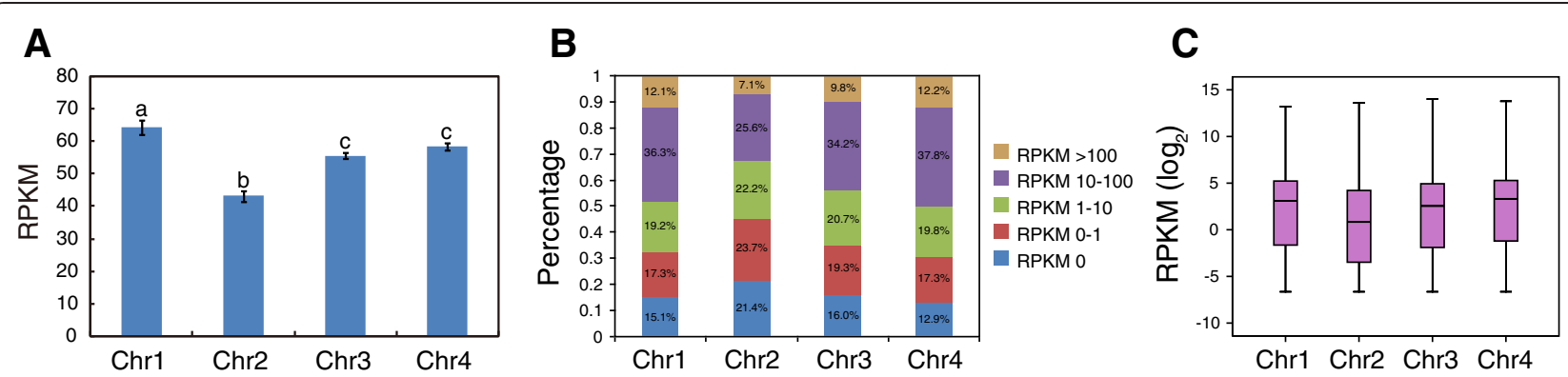

Figure 2 Evaluation of gene expression levels on the four chromosomes of Fusarium graminearum. (A) The expression levels of genes on each chromosome were quantified by the number of reads per kilobase per million reads mapped. Genes on chromosome 2 showed relative low expression levels. Different letters represent statistically significant differences $(P<0.01$, one-way ANOVA). (B) All genes of $F$. graminearum were grouped into five classes according to their RPKM values $(0,0-1,1-10,10-100,>100)$, and the proportion of each class was calculated for each chromosome. (C) Box plot analysis of gene expression on each chromosome. The expression of each gene was quantified by its RPKM value. Log2-transformed RPKM values were used to draw a box plot graph for each chromosome.

(e.g. RPKM >10) on Fgchr 2 (32.7\%) is lower than that on the other chromosomes (44-50\%). Box plot analysis also showed that relatively more weakly expressed genes $(\mathrm{RPKM}<1)$ are located on Fgchr $2(p$ value $=4.04 \mathrm{e}-39$, hypergeometric test) (Figure $2 \mathrm{C}$ ).

\section{Detailed analysis of chromosome regions with weakly expressed genes in F. graminearum}

To identify positional differences in gene expression, we divided each chromosome into $20 \mathrm{~kb}$ windows and for each window the log2-transformed read coverage was used to quantify the expression level. This analysis demonstrated that on Fgchr 2, two large regions, Nc5 (2.37 Mb $3.82 \mathrm{Mb}$ ) and Nc6 (5.44 Mb - 8.89 Mb) exhibited significantly lower expression levels (Lines B in Figure 3) ( $p$ value $<0.01$, Student's $t$-test) when compared with other parts of the same chromosome or with other chromosomes. In addition, four regions on Fgchr 1, three regions on Fgchr 3 and three regions on Fgchr 4 showed significantly lower gene expression levels. These gene expression patterns were observed for each of three biologically independent replicates (Additional file 3). Subsequently, we determined the number of genes in each of the $20 \mathrm{~kb}$ windows. Interestingly the regions with weakly expressed genes showed slightly higher gene density (Lines A in Figure 3). These data demonstrate that the expression of genes is not equally distributed across the chromosomes of F. graminearum and that the lower expression is not caused by a lower gene density in these regions.

\section{Low-expression regions coincide with non-conserved regions}

Detailed inspection showed that regions with low levels of expression correspond to non-conserved regions of F. graminearum (confer lines B and C in Figure 3). This was further analyzed by comparing genes predicted in the genome of $F$. graminearum to all the genes in
F. verticillioides using BLASTn (Additional file 4). For 9297 genes in F. graminearum, conserved genes were found in $F$. verticillioides ( $p$ value $\leq 1 \mathrm{E}-5$ ), while for 4024 genes in $F$. graminearum, no conserved ortholog in $F$. verticillioides was identified ( $p$ value $>1 \mathrm{E}-5)$. When calculating the number of non-conserved genes in each $20 \mathrm{~kb}$ window, we found that non-conserved genes are more abundant in chromosomal regions, where genes are weakly expressed (Lines $\mathrm{C}$ in Figure 3). This indicates that the regions with weakly expressed genes coincide with the non-conserved regions in F. graminearum. In addition, genes that are not expressed in nutrient-rich medium are enriched in non-conserved regions (Lines D in Figure 3).

To investigate whether non-conserved genes show lower levels of expression, the expression levels of all conserved and non-conserved genes were categorized according to their RPKM values. On each chromosome, the conserved genes showed much higher gene expression levels than non-conserved genes (Figure 4A). Furthermore, we categorized the genes of $F$. graminearum into five groups according to the $p$ value of the predicted genes in the BLAST analysis using the predicted genes of $F$. verticillioides as a reference ( $p$ value $0,0-1 \mathrm{E}-100,1 \mathrm{E}-100$ $1 \mathrm{E}-50,1 \mathrm{E}-50$ - 1E-5, >1E-5). Compared to the other three chromosomes, Fgchr 2 contains a higher proportion of non-conserved genes ( $p$ value $>1 \mathrm{E}-5$ ) (Figure 4B). In addition, a strong correlation between the degree of gene conservation and gene expression level was observed (Figure 4C), as genes with high similarity to their homologs showed relatively higher expression levels, while genes with less similarity to their homologs showed relatively lower expression levels. To specifically demonstrate this phenomenon the expression levels of all 710 transcription factors, previously identified by Son et al. [15] were analyzed. As observed for the expression levels of all genes, the expression levels of genes encoding transcription 


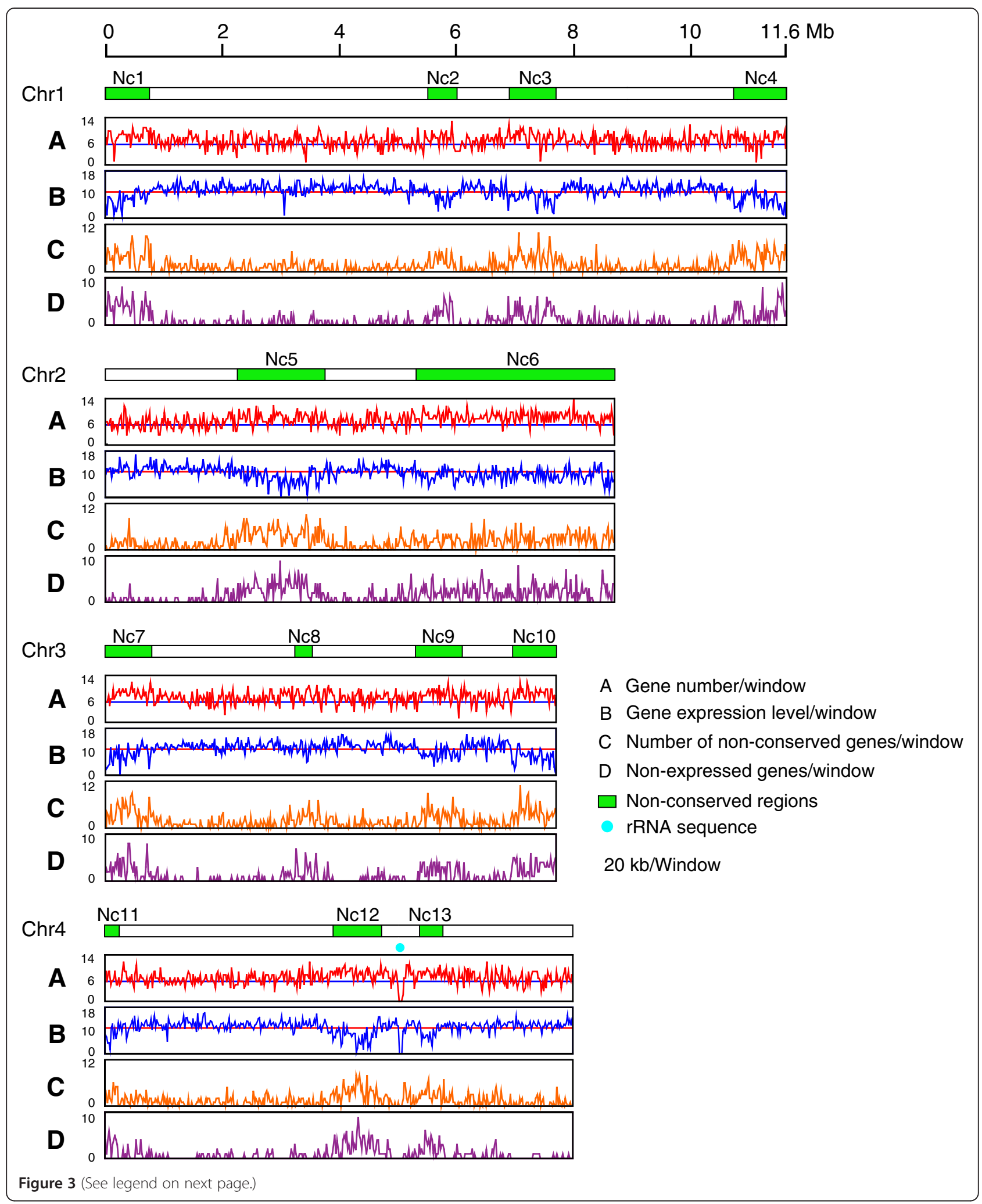


(See figure on previous page.)

Figure 3 Gene expression patterns across each of the four chromosomes of Fusarium graminearum. Each chromosome was divided into $20 \mathrm{~kb}$ windows. (A) Number of genes per window. The horizontal blue lines represent gene number of 6. (B) Gene expression levels per window. Log2-transformed read number per window was used to quantify gene expression levels. Twelve regions with low expression levels were identified on the four chromosomes, which matched non-conserved regions (except Nc8). The horizontal red lines represent an expression level of 10.

(C) Number of non-conserved genes per window. All genes predicted in F. graminearum were matched with the genes of $F$. verticillioides using BLASTn tool and genes without homologs ( $p$ value $>1 \mathrm{E}-5$ ) were selected. The number of these non-conserved genes per $20 \mathrm{~kb}$ window is shown in each chromosome of F. graminearum. (D) The number of non-expressed genes in each window of each chromosome is shown. Green boxes represent non-conserved regions in F. graminearum. The rDNA region on chromosome 4 is indicated by a blue dot.

factors with high similarity to their putative homologs in F. verticillioides were higher than genes encoding nonconserved transcription factors (Figure 4D). Furthermore, when each category of genes, grouped by the degree of similarity, was mapped on the four chromosomes of $F$. graminearum, a clear differential gene distribution pattern was observed. Conserved genes tend to cluster together as well as less conserved genes (Additional file 5). Strikingly, no conserved genes $(p$ value $=0)$ are located at one of the terminal regions of Fgchr 3.

\section{Expression of genes in non-conserved regions is highly variable in different developmental stages}

To investigate whether gene expression in non-conserved regions is invariably low, or whether it is related to a specific growth stage, RNA-Seq analysis was performed on
A

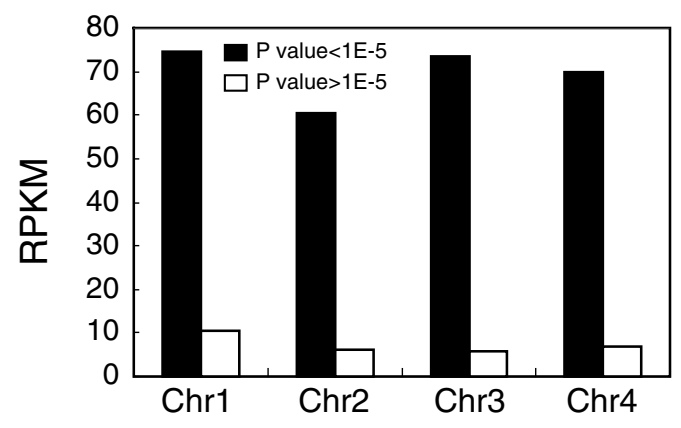

\section{B}

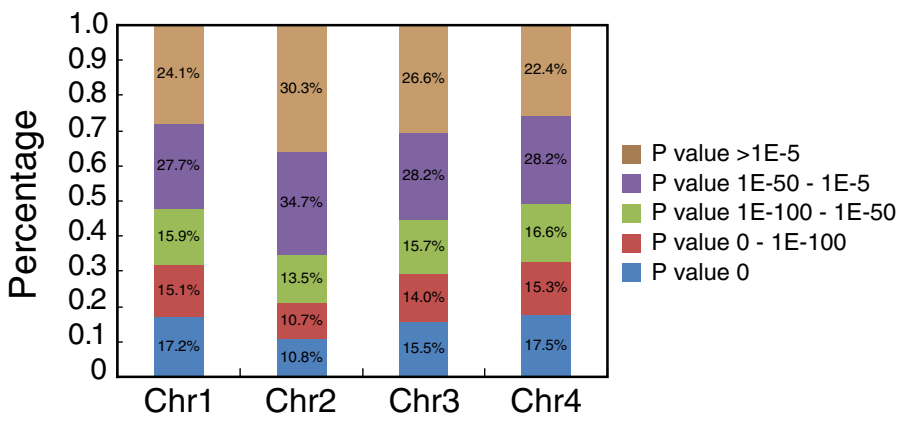

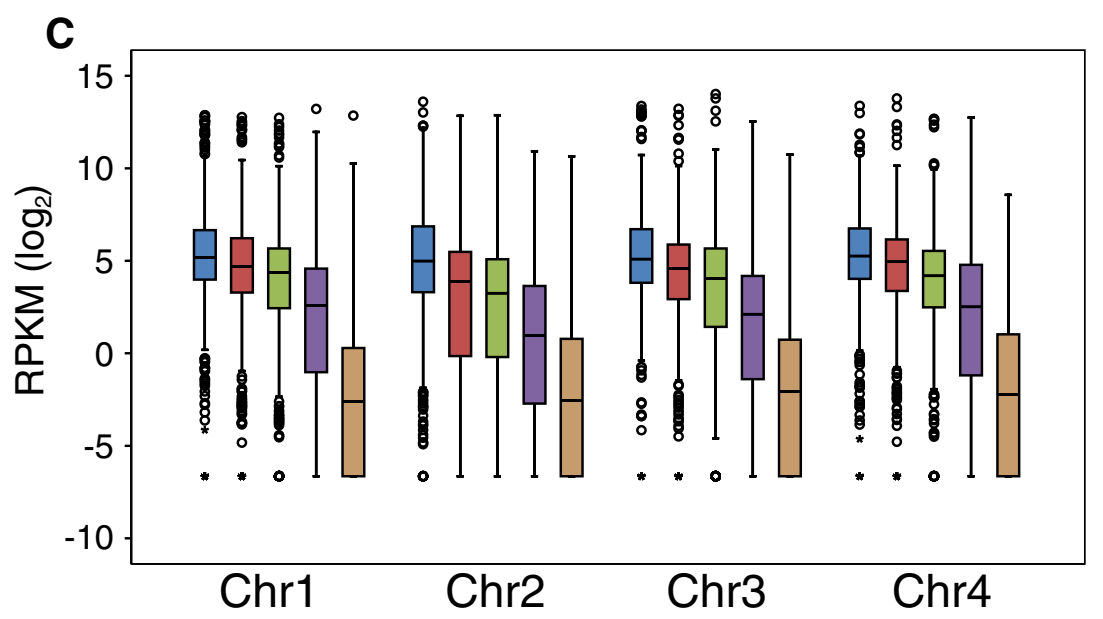

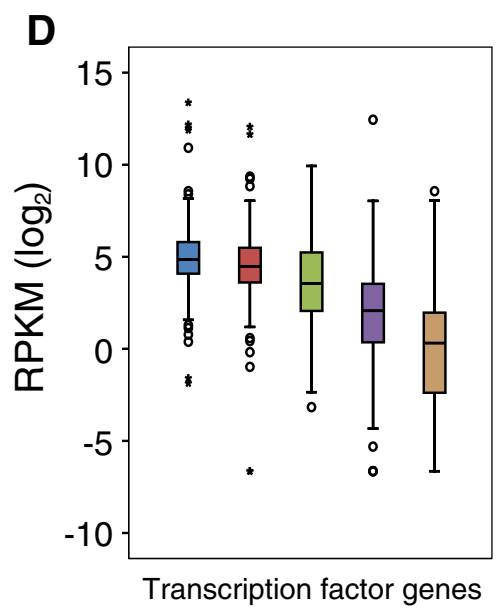

Figure 4 Gene expression levels in relation to their degree of similarity. (A) The expression levels of genes with orthologs ( $p$ value $<1 \mathrm{E}-5$ ) or genes without orthologs ( $p$ value $>1 \mathrm{E}-5$ ) in F. verticillioides were quantified by their RPKM values. (B) Genes were categorized into five groups according to their degree of similarity with those of F. verticillioides ( $p$ value $0,0-1 \mathrm{E}-100,1 \mathrm{E}-100-1 \mathrm{E}-50,1 \mathrm{E}-50-1 \mathrm{E}-5$ and $>1 \mathrm{E}-5$ ). The proportion of each category of genes on each chromosome was calculated. (C) Box plot analysis of the expression of genes on each chromosome according to their degree of similarity with those of F. verticillioides. Log2-transformed RPKM values were used to evaluate gene expression levels. (D) Box plot analysis of the expression of 710 transcription factor genes (as identified by Son et al., 2011) according to the degree of similarity with those of F. verticillioides. Log2-transformed RPKM values were used to evaluate gene expression levels. 
conidia of $F$. graminearum isolate $\mathrm{PH}-1$ produced in mung bean medium. Three biologically independent replicates were analyzed and in total 40,615,406 reads were obtained. Gene expression patterns on each chromosome of F. graminearum were analyzed with the obtained RNASeq data. Firstly, similar gene expression patterns on each chromosome of $F$. graminearum were observed for the three biological replicates (Additional file 6). Secondly, also in conidia nearly all non-conserved regions exhibited significantly lower gene expression levels than conserved regions ( $p$ value $<0.01$, Student's $t$-test). However, some parts of non-conserved regions, especially in regions Nc4 and Nc7 showed expression levels similar to conserved regions (lines B in Figure 5). In addition, we compared the gene expression levels between conidia and mycelium in each $20 \mathrm{~kb}$ window on each chromosome. Strikingly, the relative expression (e.g. expression in mycelium/expression in conidia) of genes located in conserved regions is relatively constant, while the relative expression of genes in non-conserved regions is sometimes highly variable (lines $C$ in Figure 5). Furthermore, we selected the 300 genes that exhibit the strongest up- and down-regulation between the two different developmental stages and mapped their locations on each chromosome of F. graminearum. We found that these highly induced or repressed genes are significantly abundant in non-conserved regions $(p$ value $=2.57 \mathrm{e}-49$, hypergeometric test) (lines $\mathrm{D}$ in Figure 5$)$. These data suggest that gene expression in nonconserved regions might be important for F. graminearum to respond to external stimuli or during specific phases of its life cycle, while gene expression in conserved regions are more stable and are more likely to be involved in housekeeping functions.

\section{Non-conserved regions are enriched for genes encoding secreted proteins and enzymes required for the production of secondary metabolites}

To further understand the biological relevance of nonconserved regions, we examined which categories of genes are enriched in the non-conserved regions. Firstly, we studied the distribution of all genes encoding secreted proteins in F. graminearum. Previously, Brown et al. [16] identified 574 genes encoding secreted proteins in the F. graminearum genome [16]. Distribution of these genes on each chromosome of $F$. graminearum showed that 384 locate in non-conserved regions and 190 in conserved regions (Figure 6A), suggesting that they are significantly enriched in non-conserved regions ( $p$ value $=4.32 \mathrm{e}-69$, hypergeometric test). Moreover, a higher proportion of genes encoding secreted proteins were observed on chromosome 2 ( $p$ value $=5.77 \mathrm{e}-15$, hypergeometric test $)$ (Figure 6B). Secondly, the distribution of secondary metabolite genes, including 15 polyketide synthase (PKS) genes and 19 non-ribosomal peptide (NRPS) genes [17] also appeared to be unevenly distributed: 14 PKS genes and 17 NRPS genes locate in non-conserved regions, while only one PKS gene and two NRPS genes were found in conserved regions. This indicates that genes involved in secondary metabolism are also enriched in non-conserved regions. $(p$ value $=3.09 \mathrm{e}-9$, hypergeometric test $)$. In contrast, ribosomal genes that are essential for growth are enriched in conserved regions ( $p$ value $=2.56 \mathrm{e}-14$, hypergeometric test) and fewer ribosomal genes are identified on chromosome 2 of F. graminearum (Figure 6A, C). In contrast, transcription factor-encoding genes were randomly distributed on each chromosome of F. graminearum (Figure 6A, D) ( $p$ value $=0.03$, hypergeometric test).

\section{Non-conserved regions are enriched for relocated genes}

As mentioned above, 9297 genes in F. graminearum have homologs in F. verticillioides. To further demonstrate that genes with their putative homologs in F. verticillioides are likely to be true orthologs, the homologs in F. verticillioides were aligned against the genes in F. graminearum using BLASTn and we found that 9208 gene pairs are the reciprocal best blast hits, suggesting that they are orthologs (Additional file 4). These genes can be further divided into two groups based on their collinearity with their orthologs. Most F. graminearum genes have their putative orthologs on one of the collinear chromosomes of $F$. verticillioides, while some genes have their putative orthologs located on non-collinear chromosomes of F. verticillioides. The number of genes matching to putative orthologs on each chromosome of $F$. verticillioides is presented in Table 1. For example, on Fgchr 1, 2794 genes have a putative ortholog on the collinear chromosomes $(1,5,8)$ of F. verticillioides, whereas for 187 genes, putative orthologs were shown on non-collinear chromosomes of $F$. verticillioides. We then mapped all these genes on the chromosomes of F. graminearum and found that non-conserved regions are highly enriched for these genes (Figure 7A). This indicates that non-conserved regions in F. graminearum are enriched in genes that have undergone relocation. A similar pattern was observed when comparing F. graminearum with F. oxysporum (Figure 7B). Further evidence for these relocations comes from the fact that in $355(80.5 \%)$ of these relocations a single gene was relocated, but in $46(10.4 \%)$ cases two neighbouring genes and in $40(9.1 \%)$ cases three or more neighbouring genes were relocated together. Figure $7 \mathrm{C}$ shows one example of a relocation of four neighbouring genes in F. graminearum. On Fvchr1 four genes were found between genes FVEG_01045 and FVEG_01040, whereas no genes were identified between orthologs FGSG_00567 and FGSG_00568 in F. graminearum, indicating that these four genes have been relocated to a new location in F. graminearum. BLAST analysis of the four $F$. verticillioides genes showed that their 


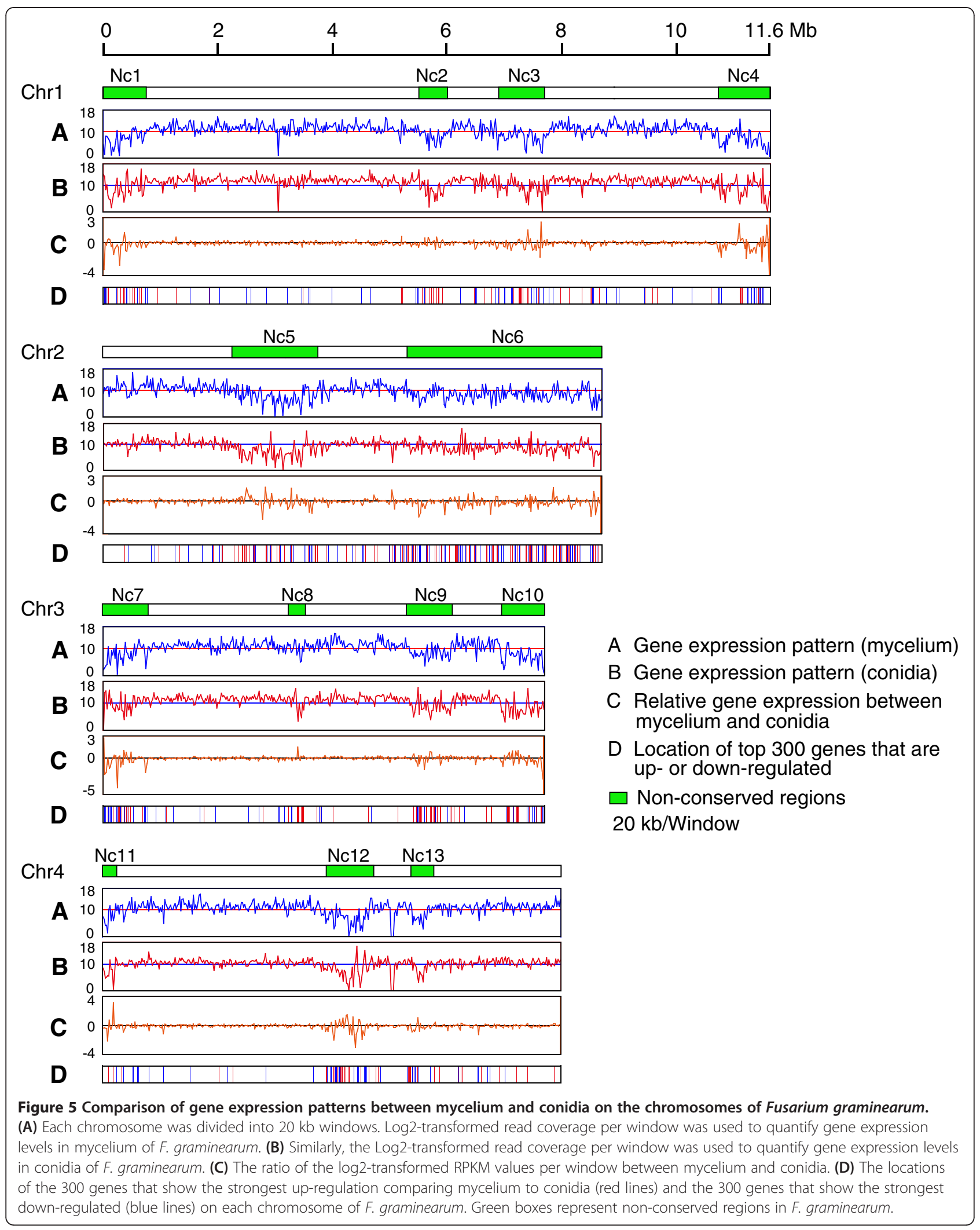




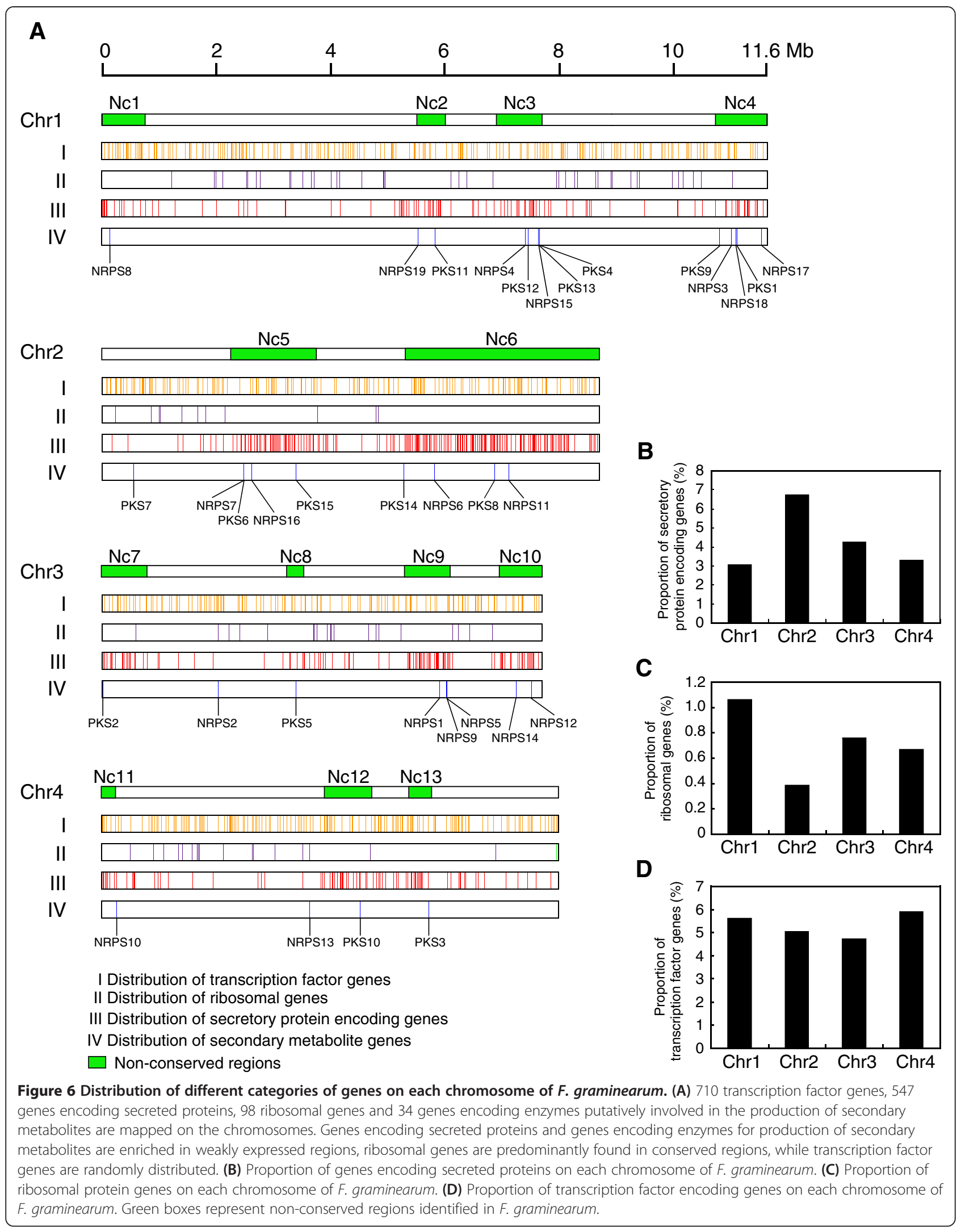


Table 1 Number of genes on each chromosome of Fusarium graminearum that have orthologs on each chromosome of F. verticillioides

\begin{tabular}{lccccccccccc}
\hline & Fvchr1 & Fvchr2 & Fvchr3 & Fvchr4 & Fvchr5 & Fvchr6 & Fvchr7 & Fvchr8 & Fvchr9 & Fvchr10 & Fvchr11 \\
\hline Fgchr1 & $\mathbf{1 4 7 8}$ & 15 & 24 & $\mathbf{7 4}$ & $\mathbf{9 5 8}$ & 17 & 13 & $\mathbf{3 5 8}$ & 12 & 19 & 13 \\
Fgchr2 & 31 & 26 & 17 & 35 & 17 & $\mathbf{7 7 2}$ & 12 & 27 & $\mathbf{5 6 8}$ & $\mathbf{3 3 3}$ & $\mathbf{2 8 1}$ \\
Fgchr3 & 24 & $\mathbf{4 8 5}$ & 14 & $\mathbf{6 8 1}$ & 18 & 7 & $\mathbf{6 2 3}$ & 72 & 17 & 15 & 20 \\
Fgchr4 & 8 & $\mathbf{6 3 2}$ & $\mathbf{1 0 3 4}$ & $\mathbf{1 5 2}$ & 8 & 11 & 103 & 68 & 20 & 14 & 18 \\
\hline
\end{tabular}

Values in bold type are the numbers of genes that have orthologs on collinear chromosomes of $F$. verticillioides, while values in regular type are the numbers of genes that have orthologs on non-collinear chromosomes of $F$. verticillioides.

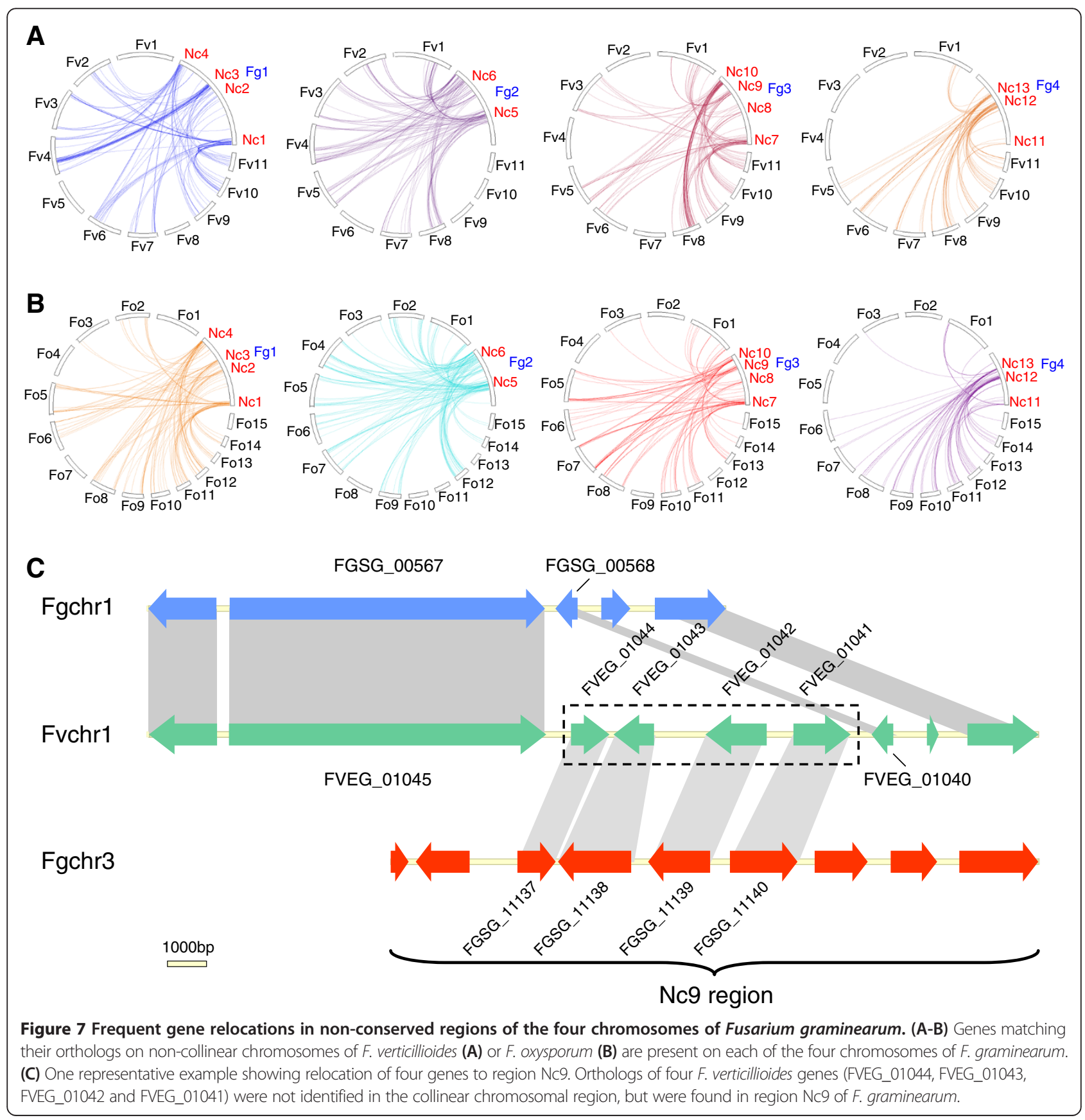


orthologs in F. graminearum are relocated to the nonconserved region Nc9 of Fgchr 3.

To understand whether genes that are relocated to nonconserved regions are also affected in expression, we compared the expression levels of relocated genes with non-relocated genes. This analysis indicated that expression of relocated genes was significantly lower than nonrelocated genes under the conditions examined (confer A and $\mathrm{B}$ in Additional file 7).

\section{Secondary metabolite gene clusters are assembled in non-conserved regions by gene relocations}

As shown above, most of secondary metabolite genes are located in non-conserved regions, and previous studies have shown that genes producing secondary metabolites are often clustered [17], but whether these secondary metabolite gene clusters are assembled by gene relocations is still unknown. Here we found that gene clusters, such as PKS1, PKS2, PKS6, PKS8 and PKS10, are probably formed by gene relocations, as genes in these clusters have orthologs that are located on different chromosomes of $F$. verticillioides. This is exemplified by the PKS2 gene cluster of which most genes have high similarity to their orthologs (Table 2). The ortholog of PKS2 gene is located on chromosome 1 of $F$. verticillioides. Other genes that are typically associated with secondary metabolite gene clusters, such as MFS monosaccharide transporter, NAD binding oxidoreductase, alcohol dehydrogenase, cytochrome P450 family protein and C6 transcription factor, have their orthologs located on the chromosomes 8, 9, 10 and 11 of $F$. verticillioides, respectively (Figure 8 and Table 2). This result suggests that secondary metabolite gene clusters could be formed by gene relocations as was found for other genes in the non-conserved regions.

\section{The origin of lineage-specific chromosomes in F. oxysporum}

F. oxysporum f. sp. lycopercisi isolate 4287 contains eleven core chromosomes and four lineage-specific (LS) chromosomes [9]. Comparison of core chromosomes of F. oxysporum with those of $F$. verticillioides through synteny analysis revealed several non-conserved regions (Additional file 1), especially at the end of chromosomes 1 and 2. To determine whether the four LS chromosomes and nonconserved regions on core chromosomes of F. oxysporum are enriched for gene relocations, we selected all genes of F. oxysporum with homologs on a non-collinear chromosome of $F$. verticillioides and assigned these genes to each chromosome of F. oxysporum. The four LS chromosomes appear to be enriched for genes that have relocated from core chromosomes (Additional file 8). Gene relocations were also supported by the observation that multiple groups of adjacent genes on LS chromosomes showed collinearity with their homologs on the chromosomes of
F. verticillioides (Additional file 9). In addition, relocation of genes was also observed in non-conserved regions of core chromosomes of $F$. oxysporum, especially in one telomere proximal region of chromosomes 1 and 2 . In addition, synteny block analysis of chromosomes in F. oxysporum was performed and we found that most of the genes present on LS chromosomes 3 and 6 are duplicated, which is consistent with previous findings [9]. Some genes on LS chromosome 14 might originate from LS chromosomes 3 or 6 . Interestingly, genes on LS chromosome 15 are duplicated from core chromosome 1 (Additional file 10), suggesting that LS chromosome 15 originally arose from core chromosome.

\section{Discussion}

Genome comparison between closely related species may help us to understand the mechanism of genome evolution and apprehend how species evolve to adapt to new environments [18-20]. Comparing the genome of Homo sapiens with the closely related species chimpanzee showed that $98.76 \%$ of the genomic sequences are similar [21], but 1,576 putative inversions were identified on the chromosomes of $H$. sapiens [22] that are likely to be involved in genome evolution. Large-scale translocations and inversions appear to have also occurred in the ascomycete Saccharomyces cerevisiae [23,24]. Here, we compared the genome of $F$. graminearum with that of two closely related species $F$. verticillioides and $F$. oxysporum. Again, a large number of translocations and inversions were identified. Next to these translocations and inversions of large chromosomal segments, non-conserved regions are commonly discovered in closely related species. For instance, synteny analysis of the genome of Aspergillus nidulans with related species $A$. fumigatus and $A$. oryzae showed that around $78 \%$ of the genome could be mapped to conserved syntenic blocks, while the remaining genomic sequences lack significant syntenic blocks [25]. In addition, syntenic analysis of 12 sequenced Drosophila species showed that on average $66 \%$ of each genome was covered by syntenic blocks [26], indicating that the location of the remaining $34 \%$ of the genome is not conserved. Comparing the genome of F. graminearum with those of $F$. verticillioides and $F$. oxysporum, thirteen non-conserved regions were identified. The presence of these non-conserved regions suggests that genome evolution has occurred unevenly across the chromosomes. Although non-conserved regions frequently occur in the genomes of many species, their origin and biological relevance are still largely unknown.

The development of RNA-Seq technology [27] allowed us to evaluate the global gene expression patterns along chromosomes [28,29]. In this study, we used RNA-Seq data obtained from the mycelium of the sequenced isolate $\mathrm{PH}-1$ of $F$. graminearum grown in nutrient-rich 
Table 2 Analysis of PKS2 secondary metabolite gene cluster

\begin{tabular}{|c|c|c|c|c|c|}
\hline \multirow[t]{2}{*}{ Num } & \multirow[t]{2}{*}{ Gene ID } & \multirow[t]{2}{*}{ Putative function } & \multicolumn{3}{|c|}{ Orthologs in Fusarium verticillioides } \\
\hline & & & Gene ID & Chromosome & $p$ value \\
\hline 1 & FGSG_12582 & Sterol 3-beta-glucosyltransferase & FVEG_00073 & Fvchr 1 & 0 \\
\hline 2 & FGSG_12583 & Putative bile acid 7-alpha protein & FVEG_00076 & Fvchr 1 & $5.51 \mathrm{E}-155$ \\
\hline 3 & FGSG_04692 & Acyltransferase & FVEG_00077 & Fvchr 1 & 0 \\
\hline 4 & FGSG_04693 & Integral membrane protein PTH11 & FVEG_00078 & Fvchr 1 & 0 \\
\hline 5 & FGSG_04694 (PKS2) & Polyketide synthase & FVEG_00079 & Fvchr 1 & 0 \\
\hline 6 & FGSG_04695 & Hypothetical protein & - & - & - \\
\hline 7 & FGSG_04696 & Hypothetical protein & - & - & - \\
\hline 8 & FGSG_04697 & Hypothetical protein & FVEG_13993 & Sc26 & 0 \\
\hline 9 & FGSG_04698 & Hypothetical protein & - & - & - \\
\hline 10 & FGSG_04699 & Hypothetical protein & - & - & - \\
\hline 11 & FGSG_04700 & MFS monosaccharide transporter & FVEG_10988 & Fvchr 9 & $9.16 \mathrm{E}-83$ \\
\hline 12 & FGSG_04701 & ARCA-like protein & - & - & - \\
\hline 13 & FGSG_04702 & NAD binding oxidoreductase & FVEG_10989 & Fvchr 9 & $1.58 \mathrm{E}-55$ \\
\hline 14 & FGSG_04703 & Alcohol dehydrogenase & FVEG_13775 & Fvchr 10 & $7.36 \mathrm{E}-61$ \\
\hline 15 & FGSG_04704 & Cytochrome P450 family protein & FVEG_07686 & Fvchr 8 & 0 \\
\hline 16 & FGSG_04705 & RTA1 like protein & - & - & - \\
\hline 17 & FGSG_04706 & RTA1 domain-containing protein & - & - & - \\
\hline 18 & FGSG_12584 & C6 transcription factor & FVEG_10599 & Fvchr 11 & $6.25 E-52$ \\
\hline
\end{tabular}

"-" represents that no ortholog was identified in F. verticillioides.

medium to investigate the gene expression pattern along whole chromosomes. Interestingly, we found two striking features in F. graminearum: (i) there is a strong correlation between the degree of gene conservation and gene expression level and (ii) genes in the non-conserved regions showed lower expression levels than genes in the conserved regions. In addition, comparing gene expression levels between conidia and mycelium, we found that the expression of genes in non-conserved regions is highly variable, while the expressions of genes in conserved regions is surprisingly stable. This indicates the expression of genes in conserved regions is less dependent on fungal development (e.g. in conidia vs. mycelium), while the expression of genes in non-conserved regions seems more developmentally regulated. Furthermore, house-keeping genes are more abundant in conserved regions, while genes required in specific developmental stages or environmental conditions are found more often in nonconserved regions. This conclusion was supported by the fact that genes encoding secreted proteins or involved in the production of secondary metabolites, which are induced under specific conditions [8,30-32], are more abundant in non-conserved regions, while ribosomal genes are mainly located in conserved regions.

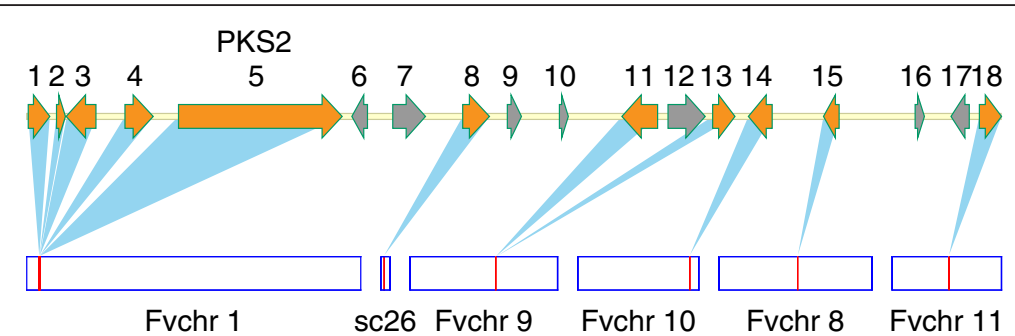

Figure 8 PKS2 gene cluster is assembled in a non-conserved region by gene relocations. The PKS2 gene and its flanking genes are matched with their orthologs in F. verticillioides. The numbers represent the genes of $F$. graminearum that are listed in Table 2. Orange colour represents genes that have orthologs, while grey colour indicates genes that have no clear orthologs in $F$. verticillioides. The boxes below represent the chromosomes of $F$. verticillioides and also the locations of orthologs on each chromosome. Sc26 is an unmapped supercontig in F. verticillioides. 
Gene enrichment in specific chromosomal regions has also been studied in other species. For instance, secondary metabolite genes are enriched in subtelomeric regions of Aspergillus species. The rapid rearrangement of subtelomeric regions may promote the rapid evolution of these genes to become species-specific attributes [25,33]. In the plant pathogenic fungus Verticillium dahliae, in planta-expressed genes are enriched in lineage-specific genomic regions that have developed by extensive chromosomal reshuffling, which was suggested to drive evolution of virulence [34]. In F. oxysporum, genes encoding secreted effectors and virulence factors are more abundant in LS chromosomes, while few house-keeping genes are identified on LS chromosomes [9]. The drivers and biological relevance of gene enrichment in specific chromosome regions are still not fully understood. One possible reason could be that clustering of genes with similar expression patterns may facilitate co-regulation of specific sets of genes under specific conditions or developmental stages. This phenomenon has been observed in many species, such as yeast, mouse and human [35-38]. Secondly, changes in gene expression levels have also been shown to be important in adaptive evolution $[39,40]$, so perhaps enrichment of genes in non-conserved regions could facilitate them to rapidly change their expression pattern.

Our data indicate that genes in non-conserved regions are weakly expressed, but how the expression of these genes is suppressed is still unknown. Previous studies have shown that gene position in the nucleus is associated with their transcriptional regulation, for instance, the nuclear periphery was considered as a zone for transcriptionally repressed genes [41]. This type of organization could also occur in F. graminearum and non-conserved regions could form specific sub-compartments of the nucleus with repressed gene expression.

The non-conserved regions occur widely, but how they are generated is still unclear. In this study, many gene relocations were identified in non-conserved regions of F. graminearum, in contrast to conserved regions. Gene relocations have been described previously in S. cerevisiae where the $D A L$ gene cluster, including six genes, was formed by gene relocations from six different loci [42]. In F. graminearum and F. sporotrichioides, trichothecene biosynthesis requires genes at three loci: the 12-gene TRI cluster, a second locus with two genes (TR1 and TRI16), and a third locus with one gene (TRI101). However, in the more distantly related species $F$. equiseti, both TRI1 and TRI101 are located within the TRI core cluster suggesting that the latter two genes have been relocated during evolution of $F$. equiseti [43]. We also showed that four successive genes were relocated to a non-conserved region in F. graminearum. However, how these genes are marked for relocation is still obscure. Based on our findings that genes with low similarity to their homologs have low expression levels (refer to Figure $4 \mathrm{C}$ ), we hypothesize that the relocated genes in non-conserved chromosome regions already had a low expression level before they were relocated. Possibly, in the three dimensional organization of chromosomes in the interphase these weakly expressed genes are in close proximity with the likewise weakly expressed non-conserved regions. Such a close proximity might facilitate targeted relocation of weakly expressed genes to one of these non-conserved regions.

In F. oxysporum, four LS chromosomes and 11 core chromosomes were identified. It was suggested that these four LS chromosomes were generated by horizontal transfer from a yet unknown fungal source [9]. Also for the dispensable chromosomes in Zymoseptoria tritici horizontal gene transfer was hypothesized [44]. Although horizontal gene transfer is one of the reasonable explanations for the origin of LS chromosomes in F. oxysporum [9], other mechanisms or events could also contribute to the formation of LS regions. In this study, the frequent gene relocations identified in non-conserved regions of $F$. graminearum drove us to hypothesize that the generation of LS chromosomes in F. oxysporum could also have included extensive gene relocations. This hypothesis was supported by the fact that around 700 genes on LS chromosomes have a homolog in F. verticillioides, and in 22 cases, two or more adjacent genes are collinear with their homologs. In addition, large part of LS chromosome 15 represents a duplication of the telomere proximal region of core chromosome 1, suggesting that it might originate from the core chromosome. Based on the studies by us and others [9], we proposed that most likely horizontal gene transfer from other fungal species and gene relocations within the species both occurred in F. oxysporum.

\section{Conclusions}

Taken together, our data demonstrate that chromosomes of F. graminearum show distinct conserved and non-conserved regions. Subsequent gene expression analysis showed that genes in these regions exhibit different expression patterns. Genes showing high and stable expression levels are more abundant in conserved regions, while genes that are induced or repressed in specific developmental stages or under different environmental conditions (mung bean medium versus liquid $\mathrm{CM}$ ) are significantly abundant in non-conserved regions. This type of genome arrangement may not only facilitate the co-regulation of specific sets of genes, but could also enable fungi to maintain on the one hand the required conservation of house-keeping genes and on the other to accelerate the evolution of species-specific genes to rapidly adapt to new environments or new hosts. Moreover, due to the selective transcription of genes in non-conserved regions, this could prevent organisms spending too much energy in transcription and 
translation of evolving genes that do not have acquired full functionality yet.

\section{Methods}

\section{RNA isolation and RNA-Seq}

Fusarium graminearum wild-type isolate $\mathrm{PH}-1$ was used in this study. To prepare conidia and mycelium for RNA isolation, PH-1 was grown in $400 \mathrm{ml}$ liquid mung bean medium for 3 days to produce conidia $\left(25^{\circ} \mathrm{C}, 200 \mathrm{rpm}\right)$. The conidia were collected by centrifugation. $10 \mathrm{e} 5$ conidia of $\mathrm{PH}-1$ were transferred to $400 \mathrm{ml}$ liquid complete medium and subsequently incubated for $30 \mathrm{~h}$ to produce mycelium $\left(25^{\circ} \mathrm{C}, 200 \mathrm{rpm}\right)$. Mycelium was harvested from liquid CM medium by filtration and grounded in liquid nitrogen using a mortar and pestle. The conidia and mycelium were used for RNA extraction using TRIzol reagent (Invitrogen, Cat. No. 15596-018) according to manufacturer's instructions. The quality of RNA was analyzed by Agilent 2100. RNA-Seq was performed according to protocols described previously [45].

\section{Analysis of gene homology}

Gene homology was evaluated in CLC genomic workbench. The gene database of $F$. graminearum, F. verticillioides and $F$. oxysporum were downloaded from Broad Fusarium Comparative Database. The gene database (fasta file format) of each Fusarium species were imported into CLC, and "BLASTn" option was used to align the genes of $F$. graminearum against the genes of $F$. verticillioides or F. oxysporum using default settings. Genes of F. graminearum were grouped according to their $p$ value after aligning with the genes of $F$. verticillioides by using BLASTn.

The identification of orthologs between Fusarium species was based on two criteria: (i) a cutoff of $p$ value of $1 \mathrm{E}-5$, and (ii) reciprocal best blast hits. For the genes in conserved chromosomal regions, synteny block analysis was also explored for the definition of orthologous relationship.

\section{Synteny block analysis}

Synteny block analysis was performed according to the program MCScanX with a small modification [46]. The gene data sets (fasta format file) of $F$. graminearum, $F$. verticillioides and $F$. oxysporum were downloaded from the Broad Fusarium database. The local blast database pools of $F$. verticillioides and $F$. oxysporum were created by using program formatdb. All genes in F. graminearum were analyzed against the gene database pool of $F$. verticillioides and $F$. oxysporum, respectively, by using BLAST tool. The BLAST results were exported in $\mathrm{m} 8$ format. Besides, gff file containing the information of the chromosome number (e.g. Fg1), gene name (e.g. FGSG_00001), start position and stop position of each gene on each chromosome of
Fusarium species was prepared. The BLAST file and gff file were imported for synteny block analysis according to the procedure described in the manual of MCScanX. The criteria for the synteny analysis are as follow: match score 50 , match size $>5$, gap_penalty -1 , overlap_window 5 , max gaps 25. Finally, two types of output (dual synteny plot and circle plot) were obtained by using two downstream programs.

\section{Analysis of relocated genes}

According to the BLASTn result, all $F$. graminearum genes with high similarity ( $p$ value $\leq 1 \mathrm{E}-5)$ in $F$. verticillioides or $F$. oxysporum were collected, from which we selected genes that have their putative orthologs on noncollinear chromosomes of $F$. verticillioides or $F$. oxysporum. The map of these genes to their orthologs was performed by using MCScanX software [46]. Furthermore, the sequences of all these putative orthologs from either $F$. verticillioides or $F$. oxysporum were collected and matched with all predicated genes in F. graminearum using BLASTn tool to identify the best hits to show that they are the bidirectional best hits.

\section{Gene expression analysis}

To evaluate gene expression along chromosomes, RNASeq reads were mapped to chromosome sequences of F. graminearum using software available in the CLC Genomics Workbench. The RNA-Seq reads were mapped to each chromosome by using "RNA-Seq analysis" option with default settings. The number of reads matched to each chromosome was calculated and subsequently the expression level of each chromosome was evaluated by using RPKM (reads per kilobase per million mapped reads) values. Similarly, to evaluate the expression of each gene, the transcript database of $F$. graminearum were imported in CLC and the expression level of each gene was evaluated by RPKM value.

To draw the gene expression level along each chromosome, we divided the chromosomes into portions of $20 \mathrm{~kb}$. The read coverage of each $20 \mathrm{~kb}$ window was calculated and $\log 2$-transformed reads coverage in each window was used to compare gene expression levels. To compare the gene expression level between conidia and mycelium, reads coverage of each window was compared and log2transformed reads coverage fold change was used to evaluate gene expression differences. The total gene number and the number of non-conserved genes in each $20 \mathrm{~kb}$ window were calculated manually based on the criteria described above.

To analyze the expression levels of conserved and nonconserved genes, the transcript sequences of conserved and non-conserved genes were collected and assembled, respectively. Also in this case the PRKM value was used to evaluate the expression levels of conserved and non- 
conserved genes. Box plot analysis of gene expression was performed by using SPSS software.

\section{Availability of supporting data}

The data sets supporting the results of this article are included within the article and its additional files. Six Illumina sequence data used in this study are available in the NCBI GEO repository (accession number GSE55477, http://www. ncbi.nlm.nih.gov/geo/query/acc.cgi?acc=GSE55477).

\section{Additional files}

Additional file 1: Synteny block analysis of Fusarium oxysporum with the genomic sequence of $F$. verticillioides. The genomic sequence of $F$. oxysporum strain 4287 was used to compare the genomic sequence of $F$. verticillioides by using program MCScanX. Eleven core chromosomes of $F$. oxysporum contain collinear chromosomes in $F$. verticillioides, while four LS chromosomes do not contain collinear chromosomes in $F$. verticillioides. Red triangles represent non-conserved regions identified on the chromosome 1 and 2 of $F$. oxysporum.

Additional file 2: Genome-wide gene expression analysis. Excel file showing the expression of all predicted genes in both conidia and mycelium of Fusarium graminearum (three biological replicates each).

Additional file 3: Gene expression pattern analysis using RNA-Seq data obtained from mycelium of $F$. graminearum. Each chromosome of $F$. graminearum was divided into $20 \mathrm{~kb}$ windows. For each window, the log2-transformed reads coverage was drawn to show gene expression patterns along each chromosome of $F$. graminearum. The gene expression patterns analyzed by three biologically independent RNA-Seq data obtained from mycelium of $F$. graminearum are shown. Green boxes represent non-conserved regions identified in F. graminearum.

Additional file 4: Putative orthologs of Fusarium graminearum genes in $F$. verticillioides and $F$. oxysporum. All predicted genes of F. graminearum were matched to their orthologs in both F. verticillioides and $F$. oxysporum by using BLASTn.

Additional file 5: Distribution of genes on each chromosome of F. graminearum according to their similarity to genes in F. verticillioides. Genes on each chromosome were divided into five groups according to their degree of similarity. Genes with a low degree of similarity to their orthologs are enriched in weakly expressed regions, while genes with a high degree of similarity to their orthologs are enriched in highly expressed regions. Green boxes represent non-conserved regions identified in F. graminearum. Note the absence of conserved genes ( $p$ value $=0$ ) at the telomeric region of $\mathrm{Chr} 3$, corresponding to $\mathrm{Nc10}$.

Additional file 6: Gene expression pattern analysis using RNA-Seq data obtained from conidia of $F$. graminearum. Each chromosome of F. graminearum was divided into $20 \mathrm{~kb}$ windows. For each window, the log2-transformed reads coverage was drawn to show gene expression patterns along each chromosome of F. graminearum. The gene expression patterns analyzed by three biologically independent RNA-Seq data obtained from the conidia of $F$. graminearum are shown. Green boxes represent non-conserved regions identified in F. graminearum.

Additional file 7: Comparison of the expression of relocated and non-relocated genes. All genes that have orthologs in F. verticillioides were divided into two groups: genes that are relocated to non-conserved regions and genes that are not relocated. Box plot analysis shows that the expression levels of relocated genes in non-conserved regions (B) are lower than non-relocated genes (A). Asterisk indicates significant difference ( $p$ value $<0.01$, Student's $t$-test)

Additional file $\mathbf{8}$ LS chromosomes in F. oxysporum are enriched for gene relocations. Genes that match their orthologs on non-collinear chromosomes of $F$. verticillioides are distributed on the chromosomes of F. oxysporum. Four LS chromosomes, Fo3, Fo6, Fo14 and Fo15, show multiple gene relocations. In addition, the telomere proximal regions of the core chromosomes, especially chromosome 1 and 2, show multiple gene relocations.

Additional file 9: Group of neighboring genes on LS chromosomes of Fusarium oxysporum that are collinear with their homologs in F. verticillioides.

Additional file 10: Synteny block analysis of each chromosome against other chromosomes in $F$. oxysporum. The genomic sequence of each chromosome was used to compare other chromosomes by using program MCScanX. Genomic sequence duplications were identified between four LS chromosomes. Remarkably, LS chromosome 15 is duplicated from the telomere proximal region of core chromosome 1.

\section{Abbreviations}

Nc: Non-conserved; RPKM: Reads per kilobase per million mapped reads; LS: Lineage-specific; SNP: Single nucleotide polymorphism.

\section{Competing interests}

The authors declare that they have no competing interests.

\section{Authors' contributions}

$C Z$ and TVdL conceived the study. CZ performed the experiments. CZ, CW, DT and TrdL analyzed the data. CZ, CW, PJGM and TvdL wrote the manuscript. All authors have interpreted the data, read and approved the manuscript.

\section{Acknowledgements}

This work was supported by grants from the National Basic Research Program of China (2011CB100700) and Chinese Academy of Sciences (SAJC201305). P. J. G. M. de Wit is supported by grants from the Royal Netherlands Academy of Arts and Sciences, the Centre for BioSystems Genomics. We would like to thank Dr. Jan-Peter Nap (Applied Bioinformatics PRT WUR) for stimulating discussion.

\section{Author details}

${ }^{1}$ Plant Research International, Wageningen, The Netherlands. ${ }^{2}$ Graduate School Experimental Plant Sciences, Wageningen University, Wageningen, The Netherlands. ${ }^{3}$ State Key Laboratory of Plant Cell and Chromosome Engineering, Institute of Genetics and Developmental Biology, Chinese Academy of Sciences, Beijing, China. ${ }^{4}$ Graduate University of Chinese Academy of Sciences, Beijing, China. ${ }^{5}$ Laboratory of Phytopathology, Wageningen University, Wageningen, The Netherlands.

Received: 16 December 2013 Accepted: 7 March 2014 Published: 13 March 2014

\section{References}

1. Bai G, Shaner G: Management and resistance in wheat and barley to fusarium head blight. Annu Rev Phytopathol 2004, 42:135-161.

2. Parry DW, Jenkinson P, Mcleod L: Fusarium Ear Blight (Scab) in Small-Grain Cereals - a Review. Plant Pathology 1995, 44:207-238.

3. Oren L, Ezrati S, Cohen D, Sharon A: Early events in the Fusarium verticillioides-maize interaction characterized by using a green fluorescent protein-expressing transgenic isolate. Appl Environ Microbiol 2003, 69:1695-1701.

4. Kistler HC, Alabouvette C, Baayen RP, Bentley S, Brayford D, Coddington A, Correll J, Daboussi MJ, Elias K, Fernandez D, Gordon TR, Katan T, Kim HG, Leslie JF, Martyn RD, Migheli Q, Moore NY, O'Donnell K, Ploetz RC, Rutherford MA, Summerell B, Waalwijk C, Woo S: Systematic Numbering of Vegetative Compatibility Groups in the Plant Pathogenic Fungus Fusarium oxysporum. Phytopathology 1998, 88:30-32.

5. Gordon TR, Martyn RD: The evolutionary biology of Fusarium oxysporum. Annu Rev Phytopathol 1997, 35:111-128.

6. Ortoneda M, Guarro J, Madrid MP, Caracuel Z, Roncero MI, Mayayo E, Di Pietro A: Fusarium oxysporum as a multihost model for the genetic dissection of fungal virulence in plants and mammals. Infect Immun 2004, 72:1760-1766.

7. Gardiner DM, McDonald MC, Covarelli L, Solomon PS, Rusu AG, Marshall M, Kazan K, Chakraborty S, McDonald BA, Manners JM: Comparative pathogenomics reveals horizontally acquired novel virulence genes in fungi infecting cereal hosts. PLoS Pathog 2012, 8:e1002952. 
8. Cuomo CA, Guldener U, Xu JR, Trail F, Turgeon BG, Di Pietro A, Walton JD, Ma LJ, Baker SE, Rep M, Adam G, Antoniw J, Baldwin T, Calvo S, Chang YL, Decaprio D, Gale LR, Gnerre S, Goswami RS, Hammond-Kosack K, Harris LJ, Hilburn K, Kennell JC, Kroken S, Magnuson JK, Mannhaupt G, Mauceli E, Mewes HW, Mitterbauer R, Muehlbauer G, et al: The Fusarium graminearum genome reveals a link between localized polymorphism and pathogen specialization. Science 2007, 317:1400-1402.

9. Ma LJ, van der Does HC, Borkovich KA, Coleman JJ, Daboussi MJ, Di Pietro A, Dufresne M, Freitag M, Grabherr M, Henrissat B, Houterman PM, Kang S, Shim WB, Woloshuk C, Xie X, Xu JR, Antoniw J, Baker SE, Bluhm BH, Breakspear A, Brown DW, Butchko RA, Chapman S, Coulson R, Coutinho PM, Danchin EG, Diener A, Gale LR, Gardiner DM, Goff S, et al: Comparative genomics reveals mobile pathogenicity chromosomes in Fusarium. Nature 2010, 464:367-373.

10. Coleman JJ, Rounsley SD, Rodriguez-Carres M, Kuo A, Wasmann CC, Grimwood J, Schmutz J, Taga M, White GJ, Zhou S, Schwartz DC, Freitag M, Ma LJ, Danchin EG, Henrissat B, Coutinho PM, Nelson DR, Straney D, Napoli CA, Barker BM, Gribskov M, Rep M, Kroken S, Molnar I, Rensing C, Kennell JC, Zamora J, Farman ML, Selker EU, Salamov A, et al: The genome of Nectria haematococca: contribution of supernumerary chromosomes to gene expansion. PLoS Genet 2009, 5:e1000618.

11. Wiemann P, Sieber CM, von Bargen KW, Studt L, Niehaus EM, Espino JJ, Huss K, Michielse CB, Albermann S, Wagner D, Bergner SV, Connolly LR, Fischer A, Reuter G, Kleigrewe K, Bald T, Wingfield BD, Ophir R, Freeman S, Hippler M, Smith KM, Brown DW, Proctor RH, Munsterkotter M, Freitag M, Humpf HU, Guldener U, Tudzynski B: Deciphering the cryptic genome: genome-wide analyses of the rice pathogen Fusarium fujikuroi reveal complex regulation of secondary metabolism and novel metabolites. PLOS Pathog 2013, 9:e1003475.

12. van der Does HC, Lievens B, Claes L, Houterman PM, Cornelissen BJ, Rep M: The presence of a virulence locus discriminates Fusarium oxysporum isolates causing tomato wilt from other isolates. Environ Microbiol 2008, 10:1475-1485

13. Houterman PM, Ma L, van Ooijen G, de Vroomen MJ, Cornelissen BJ, Takken FL, Rep M: The effector protein Avr2 of the xylem-colonizing fungus Fusarium oxysporum activates the tomato resistance protein $1-2$ intracellularly. Plant J 2009, 58:970-978.

14. Rep M, van der Does HC, Meijer M, van Wijk R, Houterman PM, Dekker HL, de Koster CG, Cornelissen BJ: A small, cysteine-rich protein secreted by Fusarium oxysporum during colonization of xylem vessels is required for 1-3-mediated resistance in tomato. Mol Microbiol 2004, 53:1373-1383.

15. Son H, Seo YS, Min K, Park AR, Lee J, Jin JM, Lin Y, Cao P, Hong SY, Kim EK, Lee SH, Cho A, Lee S, Kim MG, Kim Y, Kim JE, Kim JC, Choi GJ, Yun SH, Lim JY, Kim M, Lee YH, Choi YD, Lee YW: A phenome-based functional analysis of transcription factors in the cereal head blight fungus, Fusarium graminearum. PLOS Pathog 2011, 7:e1002310.

16. Brown NA, Antoniw J, Hammond-Kosack KE: The predicted secretome of the plant pathogenic fungus Fusarium graminearum: a refined comparative analysis. PLoS One 2012, 7:e33731.

17. Hansen FT, Sorensen JL, Giese H, Sondergaard TE, Frandsen RJ: Quick guide to polyketide synthase and nonribosomal synthetase genes in Fusarium. Int J Food Microbiol 2012, 155:128-136.

18. Paterson $\mathrm{AH}$, Freeling $\mathrm{M}$, Tang $\mathrm{H}$, Wang $\mathrm{X}$ : Insights from the comparison of plant genome sequences. Annu Rev Plant Biol 2010, 61:349-372.

19. Scally A, Dutheil JY, Hillier LW, Jordan GE, Goodhead I, Herrero J, Hobolth A Lappalainen T, Mailund T, Marques-Bonet T, McCarthy S, Montgomery SH, Schwalie PC, Tang YA, Ward MC, Xue Y, Yngvadottir B, Alkan C, Andersen LN, Ayub Q, Ball EV, Beal K, Bradley BJ, Chen Y, Clee CM, Fitzgerald S, Graves TA, Gu $Y$, Heath $P$, Heger A, et al: Insights into hominid evolution from the gorilla genome sequence. Nature 2012, 483:169-175.

20. Chimpanzee Sequencing and Analysis Consortium: Initial sequence of the chimpanzee genome and comparison with the human genome. Nature 2005, 437:69-87.

21. Navarro A, Barton $\mathrm{NH}$ : Chromosomal speciation and molecular divergence-accelerated evolution in rearranged chromosomes. Science 2003, 300:321-324.

22. Feuk L, MacDonald JR, Tang T, Carson AR, Li M, Rao G, Khaja R, Scherer SW: Discovery of human inversion polymorphisms by comparative analysis of human and chimpanzee DNA sequence assemblies. PLoS Genet 2005, 1:e56.
23. Gordon JL, Byrne KP, Wolfe KH: Mechanisms of chromosome number evolution in yeast. PLoS Genet 2011, 7:e1002190.

24. Wei W, McCusker JH, Hyman RW, Jones T, Ning Y, Cao Z, Gu Z, Bruno D, Miranda M, Nguyen M, Wilhelmy J, Komp C, Tamse R, Wang X, Jia P, Luedi P, Oefner PJ, David L, Dietrich FS, Li Y, Davis RW, Steinmetz LM: Genome sequencing and comparative analysis of Saccharomyces cerevisiae strain YJM789. Proc Natl Acad Sci USA 2007, 104:12825-12830.

25. Galagan JE, Calvo SE, Cuomo C, Ma LJ, Wortman JR, Batzoglou S, Lee SI, Basturkmen M, Spevak CC, Clutterbuck J, Kapitonov V, Jurka J, Scazzocchio C, Farman M, Butler J, Purcell S, Harris S, Braus GH, Draht O, Busch S, D'Enfert C, Bouchier C, Goldman GH, Bell-Pedersen D, Griffiths-Jones S, Doonan JH, Yu J, Vienken K, Pain A, Freitag M, et al: Sequencing of Aspergillus nidulans and comparative analysis with $A$. fumigatus and $A$. oryzae. Nature 2005, 438:1105-1115.

26. Clark AG, Eisen MB, Smith DR, Bergman CM, Oliver B, Markow TA, Kaufman TC, Kellis M, Gelbart W, lyer VN, Pollard DA, Sackton TB, Larracuente AM, Singh ND, Abad JP, Abt DN, Adryan B, Aguade M, Akashi H, Anderson WW, Aquadro CF, Ardell DH, Arguello R, Artieri CG, Barbash DA, Barker D, Barsanti P, Batterham P, Batzoglou S, Begun D, et al: Evolution of genes and genomes on the Drosophila phylogeny. Nature 2007, 450:203-218.

27. Wang Z, Gerstein M, Snyder M: RNA-Seq: a revolutionary tool for transcriptomics. Nat Rev Genet 2009, 10:57-63.

28. Nagalakshmi U, Wang Z, Waern K, Shou C, Raha D, Gerstein M, Snyder M: The transcriptional landscape of the yeast genome defined by RNA sequencing. Science 2008, 320:1344-1349.

29. Filichkin SA, Priest HD, Givan SA, Shen R, Bryant DW, Fox SE, Wong WK, Mockler TC: Genome-wide mapping of alternative splicing in Arabidopsis thaliana. Genome Res 2010, 20:45-58.

30. Kim YT, Lee YR, Jin J, Han KH, Kim H, Kim JC, Lee T, Yun SH, Lee YW: Two different polyketide synthase genes are required for synthesis of zearalenone in Gibberella zeae. Mol Microbiol 2005, 58:1102-1113.

31. Gaffoor I, Brown DW, Plattner R, Proctor RH, Qi W, Trail F: Functional analysis of the polyketide synthase genes in the filamentous fungus Gibberella zeae (anamorph Fusarium graminearum). Eukaryot Cell 2005, 4:1926-1933.

32. Voigt CA, Schafer W, Salomon S: A secreted lipase of Fusarium graminearum is a virulence factor required for infection of cereals. Plant J 2005, 42:364-375.

33. Perrin RM, Fedorova ND, Bok JW, Cramer RA, Wortman JR, Kim HS, Nierman WC, Keller NP: Transcriptional regulation of chemical diversity in Aspergillus fumigatus by LaeA. PLoS Pathog 2007, 3:e50.

34. de Jonge $\mathrm{R}$, Bolton MD, Kombrink A, van den Berg GC: Yadeta KA. Extensive chromosomal reshuffling drives evolution of virulence in an asexual pathogen. Genome Res: Thomma BP; 2013.

35. Cohen BA, Mitra RD, Hughes JD, Church GM: A computational analysis of whole-genome expression data reveals chromosomal domains of gene expression. Nat Genet 2000, 26:183-186.

36. Hurst LD, Pal C, Lercher MJ: The evolutionary dynamics of eukaryotic gene order. Nat Rev Genet 2004, 5:299-310.

37. Woo YH, Walker M, Churchill GA: Coordinated expression domains in mammalian genomes. PLoS One 2010, 5:e12158.

38. Su Al, Wiltshire T, Batalov S, Lapp H, Ching KA, Block D, Zhang J, Soden R, Hayakawa M, Kreiman G, Cooke MP, Walker JR, Hogenesch JB: A gene atlas of the mouse and human protein-encoding transcriptomes. Proc Natl Acad Sci U S A 2004, 101:6062-6067.

39. Fraser HB, Moses AM, Schadt EE: Evidence for widespread adaptive evolution of gene expression in budding yeast. Proc Natl Acad Sci USA 2010, 107:2977-2982.

40. Brawand D, Soumillon M, Necsulea A, Julien P, Csardi G, Harrigan P, Weier M, Liechti A, Aximu-Petri A, Kircher M, Albert FW, Zeller U, Khaitovich P, Grutzner F, Bergmann S, Nielsen R, Paabo S, Kaessmann H: The evolution of gene expression levels in mammalian organs. Nature 2011, 478:343-348.

41. Sexton T, Schober H, Fraser P, Gasser SM: Gene regulation through nuclear organization. Nat Struct Mol Biol 2007, 14:1049-1055

42. Wong $\mathrm{S}$, Wolfe $\mathrm{KH}$ : Birth of a metabolic gene cluster in yeast by adaptive gene relocation. Nat Genet 2005, 37:777-782.

43. Proctor RH, McCormick SP, Alexander NJ, Desjardins AE: Evidence that a secondary metabolic biosynthetic gene cluster has grown by gene relocation during evolution of the filamentous fungus Fusarium. Mol Microbiol 2009, 74:1128-1142. 
44. Goodwin SB, M'Barek SB, Dhillon B, Wittenberg AH, Crane CF, Hane JK, Foster AJ, Van der Lee TA, Grimwood J, Aerts A, Antoniw J, Bailey A, Bluhm B, Bowler J, Bristow J, van der Burgt A, Canto-Canche B, Churchill AC, Conde-Ferraez L, Cools HJ, Coutinho PM, Csukai M, Dehal P, De Wit P, Donzelli B, van de Geest HC, van Ham RC, Hammond-Kosack KE, Henrissat B, Kilian A: Finished genome of the fungal wheat pathogen Mycosphaerella graminicola reveals dispensome structure, chromosome plasticity, and stealth pathogenesis. PLoS Genet 2011, 7:e1002070.

45. Zhao C, Waalwijk C, de Wit PJ, Tang D, van der Lee T: RNA-Seq analysis reveals new gene models and alternative splicing in the fungal pathogen Fusarium graminearum. BMC Genomics 2013, 14:21.

46. Wang $Y$, Tang H, Debarry JD, Tan X, Li J, Wang X, Lee TH, Jin H, Marler B, Guo H, Kissinger JC, Paterson AH: MCScanX: a toolkit for detection and evolutionary analysis of gene synteny and collinearity. Nucleic Acids Res 2012, 40:e49.

doi:10.1186/1471-2164-15-191

Cite this article as: Zhao et al:: Relocation of genes generates nonconserved chromosomal segments in Fusarium graminearum that show distinct and co-regulated gene expression patterns. BMC Genomics 2014 15:191.

\section{Submit your next manuscript to BioMed Central and take full advantage of:}

- Convenient online submission

- Thorough peer review

- No space constraints or color figure charges

- Immediate publication on acceptance

- Inclusion in PubMed, CAS, Scopus and Google Scholar

- Research which is freely available for redistribution 